\title{
Computed tomography versus fluoroscopic guided-sacroiliac joint injection: a prospective comparative study
}

\author{
Ahmed A. A. Bessar ${ }^{1 *} \mathbb{D}$, Mohamed M. Arnaout ${ }^{2}$, Mohammad Abd Alkhalik Basha', Shady E. Shaker ${ }^{3}$, \\ Ashraf E. Elsayed ${ }^{4}$ and Manar Awad Bessar ${ }^{1}$
}

\begin{abstract}
Background: There are limited data discussing long-term pain relief and comparability of different image-guided sacroiliac joint (SIJ) injection. This study compared CT and fluoroscopic-guided SIJ injections regarding statistically and clinically significant differences in numeric pain reduction, radiation doses, and patient's satisfaction.
\end{abstract}

Methods: A prospective study conducted on 52 patients who met specific inclusion criteria of SIJ pain. A mixture of $1 \mathrm{ml}$ of $40 \mathrm{mg}$ methylprednisolone acetate diluted in $2 \mathrm{ml}$ of lidocaine $2 \%$ was injected under either CT or fluoroscopic guidance. Numeric rating score (NRS) and Oswestry disability index (ODI) were assessed and recorded for each patient before procedure and one-week, and one-, three-, six-, and 12-months after procedure. The results were compared between both groups.

Results: Analysis of NRS one-month post-procedure showed a significant decrease from baseline in both groups: $12.5 \%$ in CT group ( $p=0.002)$ and $9.5 \%$ in fluoroscopic group $(p=0.006)$. No significant difference in NRS between two groups at one- and three-months post-procedure ( $p=0.11$ and 0.1 , respectively). There was a significant difference in NRS between two groups at six- and 12-months post-procedure ( $p=0.001$ and $<0.0001$, respectively). Comparison of ODI at six-month post-procedure revealed that both groups had a statistically significant improvement $(p<0.0001)$. There was a significant difference in ODI between two groups at six-months post-procedure $(p=0.01)$.

Conclusions: CT-guided SIJ injection compares favorably with fluoroscopic guidance and offers statistically and clinically significant long-term pain relief. The use of dose reduction protocol in CT is important for decreasing the radiation dose.

Keywords: Sacroiliac joint pain, Computed tomography, Fluoroscopy, Pain management

\section{Key points}

1. CT-guided SIJ injection compares favorably with fluoroscopic guidance and provide statistically and clinically significant long-term pain relief.

\footnotetext{
*Correspondence: Ahmedawadbessar@gmail.com

${ }^{1}$ Department of Radiodiagnosis, Faculty of Human Medicine, Zagazig University, Zagazig, Egypt

Full list of author information is available at the end of the article
}

2. There was a significant difference in NRS between the two groups at six- and 12-months post-procedure $(p=0.001$ and $<0.0001$, respectively).

3. There was a significant difference in ODI between the two groups at six-months post-procedure $(p=$ $0.01)$.

4. The use of dose reduction protocol in CT is important for decreasing the radiation dose.

5. High percentage of patients in CT group were strongly satisfied with the procedure and results compared to those in fluoroscopic group. 


\section{Background}

The sacroiliac joint (SIJ) is a weight-bearing, diarthrodial joint that consists of a fibrous capsule and synovium. The superior part of the joint is ligamentous, while the inferior part is articular cartilage [1]. SIJ pain is a common etiology of low back pain (LBP) and has been reported to be a source of pain in 15 to $30 \%$ of patients with chronic LBP [1, 2]. Medical history and physical examination are not adequate to reliably diagnose those with SIJ pain [3, 4]. The patient's response to an image-guided intra-articular injection with an anesthetic is considered the current clinical standard for diagnosing SIJ pain $[1,3,5]$.

The SIJ has a complex and variable anatomy, such that local treatment can be a therapeutic and technical challenge $[6,7]$. Imaging guidance is considered the gold standard for SIJ injection [8]. Fluoroscopy, computed tomography (CT), ultrasound (US), and magnetic resonant imaging (MRI) have been recommended for image-guided procedures. Each imaging modality has an institutional preference in routine practice according to physicians' experiences and backgrounds [9-13].

The efficacy of different imaging methods to guide SIJ injections has been extensively discussed, resulting in very high success rates of up to $90 \%$ [14-18]. However, data discussing the long-term pain relief and the comparability of different image-guided techniques are limited. To report the gaps in current knowledge about the efficacy of different image-guided methods, we conducted this prospective study comparing the therapeutic outcome of CT-guided SIJ injection to that of fluoroscopic guidance.

\section{Methods}

\section{Study design}

A prospective cohort study was conducted in a group of patients with SIJ pain seeking pain relief by SIJ injection.

\section{Ethical considerations}

The Institutional Review Board approved the study. The risk and potential benefits of the procedures were explained to all patients, and written informed consent was obtained before participating in the study. The study followed the ethical principles of the Declaration of Helsinki.

\section{Study population}

The study was carried out in a tertiary health institution between January 2017 and February 2020. Initially, a total of 208 patients with chronic LBP were screened for SIJ injections. The inclusion criteria were (i) patients who had functionally limiting pain that originated from the SIJ and rated 4 or higher on the $0-10$ numeric rating score (NRS), (ii) patients more than 18 years old, (iii) patients who had failed conservative care that consisted of physical therapy or medications, and (iv) patients with failed medical therapy. Exclusion criteria were (i) patients with exaggerated pain from sciatica, and low back pain due to facet syndrome, or positive tests for piriformis syndrome $(n=98)$, (ii) pregnant patients $(n=6)$, (iii) patients refused treatment $(n=28)$, (iv) patients with bilateral SIJ dysfunction $(n=15)$. A total of 61 patients met the diagnostic criteria for SIJ pain and were eligible to participate in the study. Nine patients were lost to follow-up early during the first 6-month of the study and excluded from the final analysis. Fifty-two patients were included in the final analysis. The patients were randomly categorized into two groups: fluoroscopic group, included 29 patients who underwent fluoroscopic-guided SIJ injection, and the $\mathrm{CT}$ group included 23 patients who underwent CT-guided SIJ injection. Figure 1 illustrates the flow diagram of our study.

\section{Patient assessment}

Before injection, all patients were interviewed and subjected to clinical examination. All clinical maneuvers were performed by an independent physician, including the abduction, flexion, external rotation, sacral distraction, Gaenslen's test, sacral thrust, thigh thrust, and lateral compression. All prior radiological procedures, including previous plain radiographic examinations and MRI images, were reviewed. At presentation and before image-guided injection, a baseline NRS and Oswestry disability index (ODI) were calculated and registered for each patient. The ODI was scored out of 50 points and converted to a percentage, with higher scores indicating greater disability.

\section{Procedure}

All injections were performed in an outpatient basis by one interventional radiologist (A.A.A.B., with 10 years of experience). Injections were done unilaterally according to clinical examinations. Patients were randomized to either CT-or fluoroscopic-guided injections by one of the authors using serially numbered containers. All procedures were performed with patients in a prone position, and the area of injection was exposed. Neither patient required sedation nor preoperative pain medication. The injection was done at the lowermost inferior part of the joint. The mixture used for injection was $1 \mathrm{~mL}$ of $40 \mathrm{mg}$ of methylprednisolone acetate (Depo-Medrol; Pfizer Canada Inc., Kirkland, Quebec, Canada) diluted in $1 \mathrm{~mL}$ of $2 \%$ lidocaine. After each injection, the puncture tract 


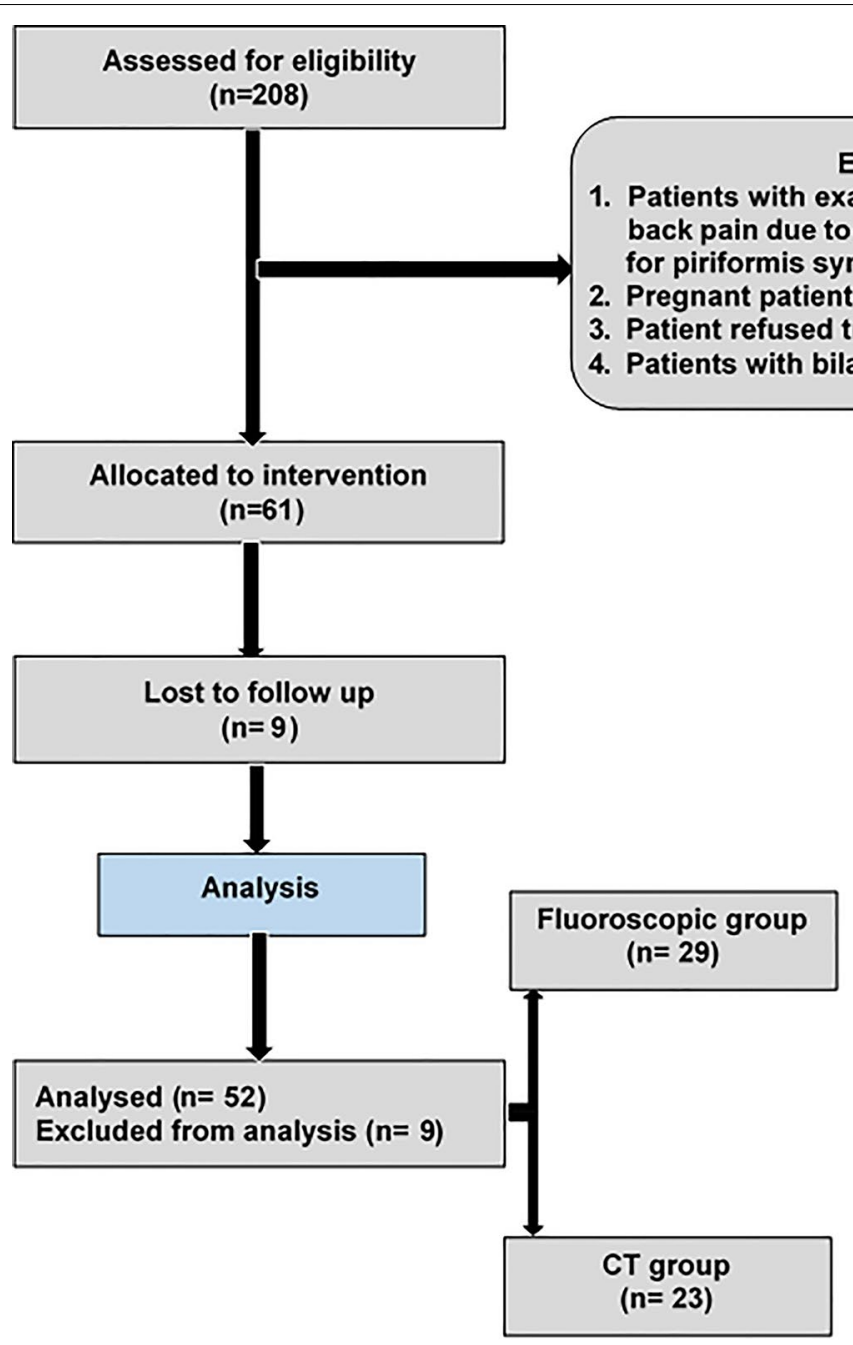

Excluded $(n=147)$

Patients with exaggerated pain from sciatica, low back pain due to facet syndrome, or positive tests pyndrome $(n=98)$.

Patient refused treatment $(n=28$ ).

Patients with bilateral SIJ dysfunction $(n=15)$.

$(n=23)$

Fig. 1 Flowchart of our study

was flushed with $0.5 \mathrm{~mL}$ lidocaine $2 \%$ while retrieving the needle to avoid spread of corticoid medication.

\section{Fluoroscopic-guided injection}

All fluoroscopic-guided injections were performed using Artis zee $\mathrm{C}$-arm (Siemens healthcare). The SIJ was detected by moving the tube to the contralateral oblique view for $15^{\circ}-45^{\circ}$ and slightly cephalad for $10^{\circ}-20^{\circ}$. This angulation made the lines of both anterior and posterior aspects of the joint overlapped, and the joint became visualized in profile view. After localization of the needle tip in the distal part of the posterior aspect of the joint and ensuring sterilization of the exposed area, a local anesthetic ( $1 \mathrm{~mL}$ of lidocaine $2 \%$ ) was injected. A 22G Quincke spinal needle, 10-cm-long, was inserted under continuous fluoroscopic guidance until the proper position in the joint space. A maximum of $0.5 \mathrm{~mL}$ of non-ionic contrast "Iohexol" (omnipaque ${ }^{\mathrm{TM}}$, GE healthcare) was injected to ensure the proper position of the needle tip that was confirmed by the upward spread of the contrast to the uppermost parts of the joint (Fig. 2).

\section{CT-guided injection}

All scans were performed using a 128-multidetector CT scanner (Ingenuity, Philips healthcare) with collimation of $128 \times 0.625$, slice thickness, and interval of $4 \mathrm{~mm}$, a pitch of 0.993 , a display field of view of $42 \mathrm{~cm}^{2}$, a matrix of $512 \times 512$, a gantry rotation of $330 \mathrm{~ms}$. A biopsy mode with reduced energy and tube current $(80 \mathrm{kV}, 50 \mathrm{mAs})$ was used. Initial scans were done, and the preferred location for injection was marked by a laser beam on the surface of the back. Each joint needed an average of 3 to 5 scans for proper needle localization. A local anesthetic ( $1 \mathrm{~mL}$ of lidocaine $2 \%$ ) was injected into the chosen point 


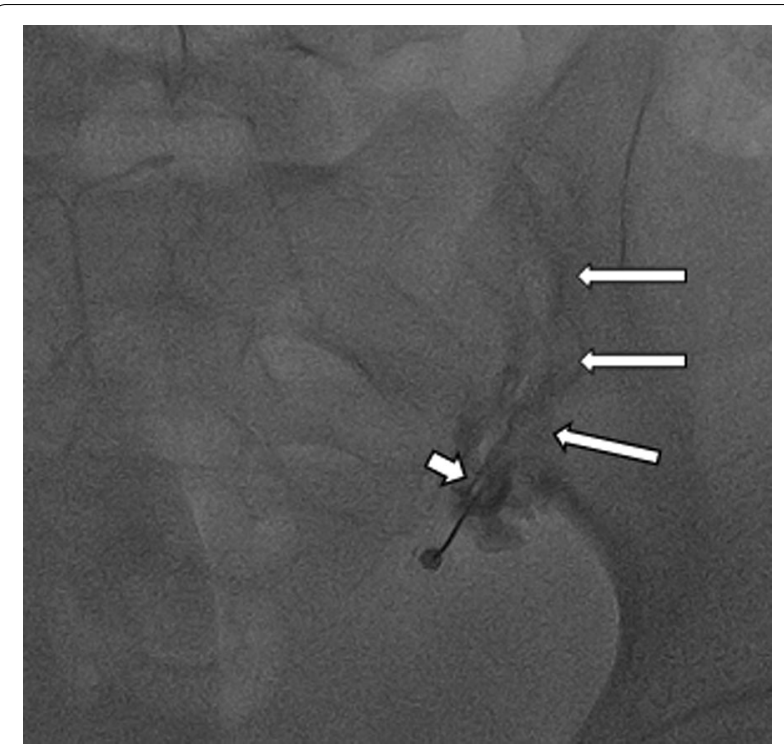

Fig. 2 A 60-year-old lady with sacroiliitis referred for SIJ injection. Fluoroscopic image obtained during SIJ injection shows the insertion of the needle tip (short arrow) within the inferior aspect of SIJ, and the linear contrast pattern (long arrows) along the course of SIJ extends away from the needle tip. Note; some extra-articular spread of contrast away from the needle tip

of injection after sterilization. A 22G Quincke spinal needle, 10-cm-long, was inserted toward the synovial part of the joint under serial cuts of $\mathrm{CT}$ (mean, $5.3 \pm 1.7$; range, 3-10). A maximum of $0.5 \mathrm{~mL}$ of non-ionic contrast "Iohexol" (omnipaque ${ }^{\mathrm{TM}}$, GE healthcare) was injected to ensure the proper position of the needle tip. After placement of the needle tip into the joint space, the mixture was injected (Fig. 3).

\section{Post-procedure}

Immediate post-procedural pain relief should be experienced in all patients to be enrolled in the study also, to confirm the absence of other sources of pain.

All patients were asked to get rest in the recovery area for two hours before going home. No postoperative oral pain medications were prescribed to monitor the degree of pain relief and prevent any bias for pain evaluation.

\section{Follow-up}

All patients were followed-up regularly, with periodical clinical visits at one week and 1, 3, 6, and 12 months after the procedure. The same physician who performed the initial clinical assessment also performed the re-examination with the same clinical maneuvers. The postoperative follow-up included a clinical examination of SIJ pain and its degree of relief, NSR, and ODI. The NRS and ODI values were reported at each visit and compared with the baseline values. The percent reduction was calculated using the formula (pre-procedure-post-procedure)/preprocedure $\times 100$. The total radiation dose was calculated automatically for each patient. The total duration of the procedure from area exposure until the end of the procedure was calculated for each patient. Patient satisfaction for the procedure was assessed on the day of the procedure, and patient satisfaction for the results of the procedure was evaluated after six months. Patient satisfaction was nominated in terms of strongly satisfied, mildly

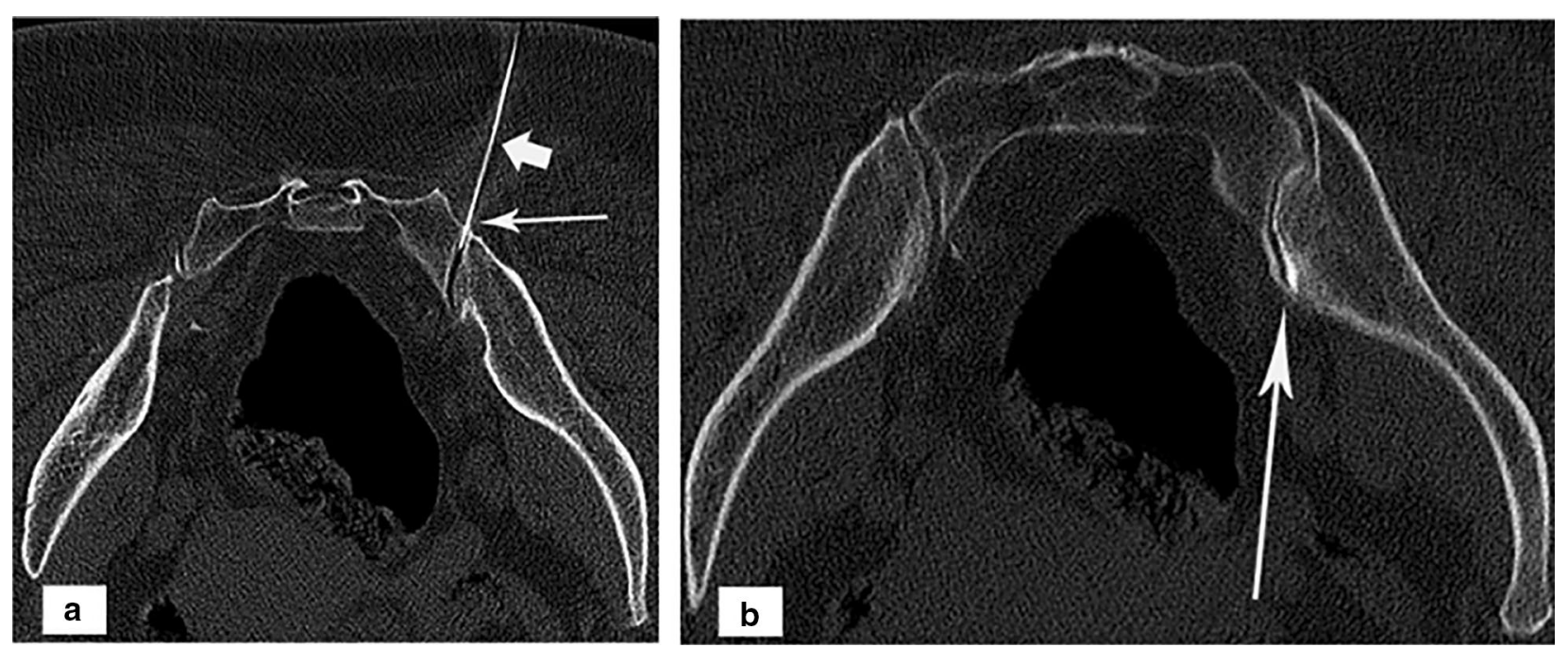

Fig. 3 A 50-year-old lady with sacroiliitis referred for SIJ injection. a A non-enhanced CT (bone window) lower cut on SIJ shows the synovial part of the joint (long arrow) where the needle (short arrow) is inserted. b A contrast-enhanced CT (bone window) upper cut reveals contrast (long arrow) within the anterior synovial part of the joint. No contrast in the posterior fibrous part of the joint 
satisfied, and not satisfied according to the patients' own words and expressions.

\section{Statistical analysis}

The data obtained were analyzed using MedCalc version 11.1. The means and standard deviations were calculated for quantitative data, and numbers and percentages were calculated for categorical data. Pre- and post-procedure results were compared using a paired sample $t$-test and Mann-Whitney $U$-test when appropriate. Categorical variables were analyzed using the Chi-square test or Fisher's exact test. A $p$ value $<0.05$ was accepted as significant.

\section{Results}

\section{Patients}

A total of 52 patients with SIJ pain (29 males and 23 females; mean age, $46 \pm 12.1$ years; range, $30-67$ years) were included in the final analysis. The basic demographic data of the patients are described in Table 1. No significant differences between the two groups in terms of pre-procedure NRS, limitation of physical function as measured by ODI, baseline characteristics, or the duration of pain before the procedure. Right-sided SIJ injection was performed in 28 patients, and left-sided injection was performed in 24 patients. All procedures were successfully completed without any immediate or delayed complications. In the fluoroscopic group, there was difficult localization of the joint space in five patients with advanced osteophytosis, three patients with complex anatomy, and two obese patients. In those patients, we increased the amount of contrast to access the joint.

\section{Comparison of clinical outcomes between the two groups}

Table 2 compared between both groups regarding outcome measures. Analysis of NRS one-month post-procedure showed a significant decrease from the baseline in both groups: $12.5 \%$ in the CT group $(p=0.002)$ and $9.5 \%$ in the fluoroscopic group $(p=0.006)$. Although there was a statistically significant improvement in both groups, there was no clinically significant difference in NRS between the two groups at one- and 3-months postprocedure $(p=0.11$ and 0.1 , respectively). There was a statistically significant difference in NRS between the two groups at six- and 12-months post-procedure $(p=0.001$ and $<0.0001$, respectively). Comparison of ODI at sixmonth post-procedure revealed that both groups had a statistically significant improvement (reduction by $51.3 \%$ and $35.4 \%$, respectively, $p<0.0001$ ). There was a significant difference in ODI between the two groups at six-months post-procedure $(p=0.01)$. The number of patients with ODI below 20 (minimal disability) was 5 in the $\mathrm{CT}$ group, whereas none in the fluoroscopic group had ODI below 20. The number of patients with ODI between 21 and 40 (moderate disability) was 16 in the CT group and 13 in the fluoroscopic group. Regarding the total duration of the procedure, there was no significant difference between the two groups (13.8 vs. $14.1 \mathrm{~min}$, $p=0.5)$. Regarding the radiation doses, no significant differences were observed between the two groups (13.2 vs. $14.5 \mathrm{mGycm}^{2}, p=0.09$ ).

\section{Comparison of overall patient satisfaction between both groups}

Eighteen (78.3\%) patients were strongly satisfied with the CT procedure compared to $10(34.5 \%)$ patients in the fluoroscopic group. Also, 14 (60.9\%) patients were

Table 1 Baseline patients' data

\begin{tabular}{|c|c|c|c|c|}
\hline & $\begin{array}{l}\text { All patients } \\
(n=52)\end{array}$ & $\begin{array}{l}\text { Fluoroscopic group } \\
(n=29)\end{array}$ & $\begin{array}{l}\text { CT group } \\
(n=23)\end{array}$ & $P$ value \\
\hline Age & $46 \pm 12.1(30-67)$ & $44.4 \pm 11.3(32-60)$ & $48.2 \pm 13.9(30-67)$ & 0.56 \\
\hline Sex, $n(\%)$ & & & & 0.79 \\
\hline Male & $29(55.8)$ & $15(51.7)$ & $14(60.9)$ & \\
\hline Female & $23(44.2)$ & $14(48.3)$ & $9(39.1)$ & \\
\hline $\mathrm{BMI}\left(\mathrm{kg} / \mathrm{m}^{2}\right)$ & $26.9 \pm 7.5(20.2-34.8)$ & $27.3 \pm 8.2(21.5-33.1)$ & $25.9 \pm 6.5(20.2-34.8)$ & 0.58 \\
\hline Side of pain, $n(\%)$ & & & & 0.57 \\
\hline Right & $28(53.8)$ & $17(58.6)$ & $11(47.8)$ & \\
\hline Left & $24(46.2)$ & $12(41.4)$ & $12(52.2)$ & \\
\hline Duration of pain (years) & $4.9 \pm 3.5(1-12)$ & $5.4 \pm 3.7(2-12)$ & $4.3 \pm 3.4(1-10)$ & 0.61 \\
\hline NRS & $7.3 \pm 0.94(5-9)$ & $7.4 \pm 0.91(6-9)$ & $7.2 \pm 0.98(5-9)$ & 0.45 \\
\hline ODI & $59.5 \pm 16.1(30-90)$ & $59.0 \pm 17.0(30-90)$ & $60.2 \pm 15.2(35-85)$ & 0.79 \\
\hline
\end{tabular}

Unless otherwise indicated, data are mean \pm standard deviation and data in parentheses are range. $C T=$ computed tomography; NRS $=$ Numerical Rating Score; $\mathrm{ODI}=$ Oswestry Disability Index 
Table 2 Comparison of outcome measures between the two groups

\begin{tabular}{|c|c|c|c|}
\hline & $\begin{array}{l}\text { Fluoroscopic group } \\
(n=29)\end{array}$ & $\begin{array}{l}\text { CT group } \\
(n=23)\end{array}$ & $P$ value \\
\hline \multicolumn{4}{|l|}{ NRS } \\
\hline Pre-procedure & $7.4 \pm 0.91(6-9)$ & $7.2 \pm 0.98(5-9)$ & 0.45 \\
\hline Post-procedure 1 week & $7.2 \pm 0.86(5-8)$ & $6.7 \pm 0.9(5-8)$ & 0.98 \\
\hline Post-procedure 1 month & $6.7 \pm 0.94(5-8)$ & $6.3 \pm 0.82(5-7)$ & 0.11 \\
\hline Post-procedure 3 months & $5.6 \pm 0.49(5-6)$ & $5.3 \pm 0.82(4-7)$ & 0.1 \\
\hline Post-procedure 6 months & $4.3 \pm 0.89(3-6)$ & $3.5 \pm 0.73(2-5)$ & 0.001 \\
\hline Post-procedure 12 months & $2.8 \pm 0.99(1-4)$ & $1.6 \pm 0.65(1-3)$ & $<0.0001$ \\
\hline \multicolumn{4}{|l|}{ ODI } \\
\hline Pre-procedure & $59.0 \pm 17.0(30-90)$ & $60.2 \pm 15.2(35-85)$ & 0.79 \\
\hline Post-procedure 6 months & $38.1 \pm 12.7(20-75)$ & $29.3 \pm 11.2(15-60)$ & 0.01 \\
\hline Duration of the procedure (minutes) & $14.1 \pm 3.2(10-20)$ & $13.8 \pm 5.4(10-24)$ & 0.5 \\
\hline Total radiation dose $\left(\mathrm{mGycm}{ }^{2}\right)$ & $13.2+2(6.7-16)$ & $14.5 \pm 2.5(13-25)$ & 0.09 \\
\hline
\end{tabular}

Data are mean \pm standard deviation. Data in parentheses are range. $\mathrm{CT}=$ computed tomography; $\mathrm{NRS}=\mathrm{Numerical}$ Rating Score; ODI = Oswestry Disability Index

Table 3 Overall patient satisfaction

\begin{tabular}{llll}
\hline & $\begin{array}{l}\text { Fluoroscopic group } \\
(n=29)\end{array}$ & $\begin{array}{l}\text { CT group } \\
(n=23)\end{array}$ & P value \\
\hline $\begin{array}{l}\text { For procedure } \\
\text { Strongly satisfied }\end{array}$ & $10(34.5)$ & $18(78.3)$ & 0.004 \\
$\begin{array}{l}\text { Mildly satisfied } \\
\text { Not satisfied }\end{array}$ & $19(65.5)$ & $5(21.7)$ & \\
$\begin{array}{l}\text { For the result } \\
\text { Strongly satisfied }\end{array}$ & $4(13.8)$ & 0 & 0.0004 \\
Mildly satisfied & $15(51.7)$ & $14(60.9)$ & \\
Not satisfied & $10(34.5)$ & $2(8.7)$ & \\
\hline
\end{tabular}

Data are number of patients. Data in parentheses are percentages. $\mathrm{CT}=$ computed tomography

strongly satisfied with the CT results compared to 4 (13.8\%) patients in the fluoroscopic group (Table 3).

\section{Discussion}

The main objective of any image-guided techniques for SIJ injection is to relieve pain rapidly and safely. In this study, we examined two image-guided techniques-the $\mathrm{CT}$ and fluoroscopic-guided injection. Although the efficacy of these techniques and their outcomes has been excessively investigated [19-24], up to date, the comparison between CT and fluoroscopy-guided SIJ injection has not been tested. The present study prospectively compared CT-guided SIJ injection and fluoroscopic guidance regarding the statistically and clinically significant differences in numeric pain reduction.

When an accurate diagnosis is of high significance, the accuracy of the injection is crucial. However, in clinical practice, the final therapeutic outcome may be more significant than the accuracy of the injection [25].
In comparing $\mathrm{CT}$ to fluoroscopically guided SIJ injection, our study found a statistically significant improvement with no clinically significant difference in NRS at one-week and one- and three-months post-procedure $(p=0.98,0.11$, and 0.1 , respectively). However, at sixand 12-months, there was a statistically significant difference in NRS between two groups $(p=0.001$ and $<0.0001$, respectively). Also, at six months, a statistically significant difference was observed in ODI between the two groups $(p=0.01)$.

The number of patients with ODI below 20 (minimal disability) was 5 in the CT group, whereas none in the fluoroscopic group had ODI below 20. The number of patients with ODI between 21 and 40 (moderate disability) was 16 in the CT group and 13 in the fluoroscopic group. Therefore, the CT-guided injection provided a long-term pain reduction compared to fluoroscopic guidance. These findings support the previous literature [22, 23], which has reported long-lasting effects of CT-guided injections in patients with SIJ pain. Also, Dussault et al. [9] found that $97 \%$ of fluoroscopically guided SIJ injections were intra-articular. However, it is difficult in some cases to place the needle into the joint under fluoroscopic guidance. In such cases, CT guidance can facilitate proper needle placement.

Based on our findings, which confirm those of previously published studies [22-24] and in view of the significant performance of CT-guided injection on prolonged SIJ pain reduction, our data support that CT-guided injection is potentially slightly better with long-term pain relief of SIJ pain than fluoroscopic guidance. The long-term pain relief by CT-guided injection in our study could be attributed to the injection of all mixtures in the intra-articular space. In contrast, 
there was a loss of some of the mixture during injection under fluoroscopic guidance due to difficult localization of the joint space in patients with advanced osteophytosis $(n=5)$ or obese patients $(n=2)$, or due to the complexity of the anatomy $(n=3)$.

Stoeckelhuber et al. [26] stated that although the fluoroscopy and CT are regarded as well-established methods to monitor the interventional treatment of SIJ pain, their radiation exposure is a disadvantage to both the interventional radiologist and younger patients. Also, Artner et al. [27] reported that despite accurate intra-articular injection in CT-guidance, radiation exposure persists as a crucial problem. Shepherd et al. [28] reported general radiation doses of $199 \mathrm{mGycm}^{2}$, and Schmid et al. [29] estimated average radiation doses of 100.7 to $235.3 \mathrm{mGycm}^{2}$ for conventional CTguided spinal interventions. In our study, no significant difference was observed between the two groups regarding radiation dose $(p=0.09)$ because we used the dose reduction protocol in CT-guided injection with proper needle position in the scout series to limit the planning axial images. The application of dose reduction protocols that diminished radiation exposure by more than $90 \%$ has been reported in a study performed by Artner et al. [27], which achieved a mean radiation dose of $4.6 \mathrm{mGycm}^{2}$ for CT-guided SIJ injection.

Our study revealed that $78.3 \%$ of patients in CT group were strongly satisfied with the procedure compared to $34.5 \%$ of patients in fluoroscopic group $(p=0.004)$. As well as, $60.9 \%$ of patients in CT group were strongly satisfied with the result of the procedure compared to $13.8 \%$ of patients in fluoroscopic group $(p=0.0004)$.

This study had limitations. First, small sample size in each arm of the study. Second, the effect of extraarticular or combined intra- and extra-articular injections to control pain form SIJ dysfunction in short- and long-term follow-up visits were not performed. Third, no comparison regarding the accuracy of each procedure. Fourth, the exclusion criteria were very strict and may limit the generalizability of the results to many practices. So further studies comparing both techniques in all patients are recommended. Fifth, the multifactorial nature of LBP with a significant proportion of pain from arthropathies in facet joints at the above levels, radiculopathy or spinal instability. Sixth, the lack of pre-procedural provocative test to measure the potential response to either fluoroscopic or CT-guided injection. Seventh, the subjective way performed in most cases to measure the degree of pain relief according to patient's words. Finally, the extra-articular spread of injectate in fluoroscopic guided interventions and the inability to localize the needles properly in some patients under X-ray guidance could be disadvantages.

\section{Conclusions}

CT-guided SIJ injection compares favorably with fluoroscopic guidance and offers statistically and clinically significant long-term pain relief. Both techniques have similar radiation doses. However, the use of dose reduction protocols in CT is important for decreasing the radiation dose. The CT-guided injection can become a widely accepted procedure for the treatment of SIJ pain. Further studies are still required to establish the long-lasting efficacy and safety of CT-guided SIJ injection compared to other image-guided techniques.

\section{Abbreviations \\ CT: Computed tomography; LBP: Low back pain; NRS: Numeric rating score; ODI: Oswestry disability index; SIJ: Sacroiliac joint.}

\section{Acknowledgements}

The authors thank all staff members and colleagues in the Radiology Department, Zagazig University, for their helpful cooperation.

\section{Authors' contributions}

Guarantor of integrity of the entire study was MB. Study concepts and design were contributed by $A A A B, M B, M A B$, and $A E E$. Literature research was contributed by AAAB, MMA, and SES. Clinical studies were contributed by MMA and SES. Experimental studies/data analysis were contributed by MAB, AB, MMA, and AEE. Statistical analysis was contributed by MB. Manuscript preparation was contributed by MB and AAAB. Manuscript editing was contributed by MB and AAAB. All authors read and approved the final manuscript.

Funding

The authors declare that this work has not received any funding.

Availability of data and materials

The datasets used and/or analyzed during the current study are available from the corresponding author on reasonable request.

\section{Declarations}

Ethics approval and consent to participate

Institutional review board approval was obtained. Written informed consent was obtained from all patients and archived in hospital files.

\section{Consent for publication}

Not applicable.

\section{Competing interests}

The authors declare that they have no competing interests.

\section{Author details}

${ }_{1}^{1}$ Department of Radiodiagnosis, Faculty of Human Medicine, Zagazig University, Zagazig, Egypt. ${ }^{2}$ Department of Neurosurgery, Faculty of Human Medicine, Zagazig University, Zagazig, Egypt. ${ }^{3}$ Department of Internal Medicine, Faculty of Human Medicine, Zagazig University, Zagazig, Egypt. ${ }^{4}$ Department of Anesthesia, Surgical Intensive Care and Pain Medicine, Faculty of Human Medicine, Zagazig University, Zagazig, Egypt.

Received: 20 November 2020 Accepted: 3 March 2021

Published online: 18 March 2021

\section{References}

1. Schwarzer AC, Aprill CN, Bogduk N (1995) The sacroiliac joint in chronic low back pain. Spine (Phila Pa 1976) 20:31-37 
2. Cohen SP, Chen Y, Neufeld NJ (2013) Sacroiliac joint pain: a comprehensive review of epidemiology, diagnosis and treatment. Expert Rev Neurother 13:99-116

3. Dreyfuss P, Michaelsen M, Pauza K, McLarty J, Bogduk N (1996) The value of medical history and physical examination in diagnosing sacroiliac joint pain. Spine (Phila Pa 1976) 21:2594-2602

4. Dreyfuss P, Dryer S, Griffin J, Hoffman J, Walsh N (1994) Positive sacroiliac screening tests in asymptomatic adults. Spine (Phila Pa 1976) 19:1138-1143

5. Maigne JY, Aivaliklis A, Pfefer F (1996) Results of sacroiliac joint double block and value of sacroiliac pain provocation tests in 54 patients with low back pain. Spine (Phila Pa 1976) 21:1889-1892

6. Hermann KG, Braun J, Fischer T, Reisshauer H, Bollow M (2004) Magnetic resonance tomography of sacroiliitis: anatomy, histological pathology, MR-morphology, and grading. Radiologe 44:217-228

7. Hermann KG, Bollow M (2014) Magnetic resonance imaging of sacroiliitis in patients with spondyloarthritis: correlation with anatomy and histology. Rofo 186:230-237

8. Rosenberg JM, Quint TJ, de Rosayro AM (2000) Computerized tomographic localization of clinically-guided sacroiliac joint injections. Clin J Pain 16:18-21

9. Dussault RG, Kaplan PA, Anderson MW (2000) Fluoroscopy-guided sacroiliac joint injections. Radiology 214:273-277

10. Bollow M, Braun J, Taupitz M et al (1996) CT-guided intraarticular corticosteroid injection into the sacroiliac joints in patients with spondyloarthropathy: indication and follow-up with contrast-enhanced MRI. J Comput Assist Tomogr 20:512-521

11. Pereira PL, Günaydin I, Trübenbach J et al (2000) Interventional MR imaging for injection of sacroiliac joints in patients with sacroiliitis. AJR Am J Roentgenol 175:265-266

12. Reach JS, Easley ME, Chuckpaiwong B, Nunley JA II (2009) Accuracy of ultrasound guided injections in the foot and ankle. Foot Ankle Int 30:239-242

13. Chen CP, Wong AM, Hsu CC et al (2010) Ultrasound as a screening tool for proceeding with caudal epidural injections. Arch Phys Med Rehabil 91:358-363

14. Maugars Y, Mathis C, Berthelot JM, Charlier C, Prost A (1996) Assessment of the efficacy of sacroiliac corticosteroid injections in spondylarthropathies: a double-blind study. Rheumatology 35:767-770

15. Günaydin IL, Pereira PL, Daikeler TH, Mohren MA, Trübenbach JO, Schick FR, Kanz LO, Kötter I (2000) Magnetic resonance imaging guided corticosteroid injection of the sacroiliac joints in patients with therapy resistant spondyloarthropathy: a pilot study. J Rheumatol 27:424-428

16. Fritz J, König CW, Günaydin I, Clasen S, Kastler B, Kötter I, Claussen CD, Pereira PL (2005) Magnetic resonance imaging-guided corticosteroidinfiltration of the sacroiliac joints: pain therapy of sacroiliitis in patients with ankylosing spondylitis. RoFo 177:555-563

17. Fischer T, Biedermann T, Hermann KG, Diekmann F, Braun J, Hamm B, Bollow M (2003) Sacroiliitis in children with spondyloarthropathy: therapeutic effect of CT-guided intra-articular corticosteroid injection. RoFo 175:814-821

18. Braun J, Bollow M, Seyrekbasan F, Häberle HJ, Eggens U, Mertz A, Distler A, Sieper J (1996) Computed tomography guided corticosteroid injection of the sacroiliac joint in patients with spondyloarthropathy with sacroiliitis: clinical outcome and follow-up by dynamic magnetic resonance imaging. J Rheumatol 23:659-664

19. Khuba S, Agarwal A, Gautam S, Kumar S (2016) Fluoroscopic sacroiliac joint injection: is oblique angulation really necessary. Pain Physician 19:E1135-1138

20. Nacey NC, Patrie JT, Fox MG (2016) Fluoroscopically guided sacroiliac joint injections: comparison of the effects of intraarticular and periarticular injections on immediate and short-term pain relief. AJR Am J Roentgenol 207:1055-1061

21. Hartung W, Ross CJ, Straub R, Feuerbach S, Schölmerich J, Fleck M, Herold T (2010) Ultrasound-guided sacroiliac joint injection in patients with established sacroiliitis: precise IA injection verified by MRI scanning does not predict clinical outcome. Rheumatology 49:1479-1482

22. Althoff CE, Bollow M, Feist E, Marticorena-Garcia SR, Eshed I, Diekhoff T, Hamm B, Hermann KG (2015) CT-guided corticosteroid injection of the sacroiliac joints: quality assurance and standardized prospective evaluation of long-term effectiveness over six months. Clin Rheumatol 34:1079-1084

23. Braun J, Bollow M, Seyrekbasan F, Häberle HJ, Eggens U, Mertz A, Distler A, Sieper J (1996) Computed tomography guided corticosteroid injection of the sacroiliac joint in patients with spondyloar-thropathy with sacroiliitis: clinical outcome and follow-up by dynamic magnetic resonance imaging. J Rheumatol 23:659-664

24. Cui Y, Xiao Z, Shuxia W, Zhenjun Z, Hengguo Z, Liangyi F, Weicheng G, Li L, Guangfeng Z, Yunzhen S, Guangfu D (2010) Computed tomography guided intra-articular injection of etanercept in the sacroiliac joint is an effective mode of treatment of ankylosing spondylitis. Scand J Rheumatol 39:229-232

25. Zheng P, Schneider BJ, Yang A, McCormick ZL (2019) Image-guided sacroiliac joint injections: an evidence-based review of best practices and clinical outcomes. PM R 11:S98-104

26. Stoeckelhuber BM, Leibecke T, Schulz E, Melchert UH, Bergmann-Koester CU, Helmberger T, Gellissen J (2005) Radiation dose to the radiologist's hand during continuous CT fluoroscopy-guided interventions. Cardiovasc Intervent Radiol 28:589-294

27. Artner J, Cakir B, Reichel H, Lattig F (2012) Radiation dose reduction in CT-guided sacroiliac joint injections to levels of pulsed fluoroscopy: a comparative study with technical considerations. J Pain Res 5:265

28. Shepherd TM, Hess CP, Chin CT, Gould R, Dillon WP (2011) Reducing patient radiation dose during CT-guided procedures: demonstration in spinal injections for pain. AJNR Am J Neuroradiol 32:1776-1782

29. Schmid G, Schmitz A, Borchardt D, Ewen K, von Rothenburg T, Koester O, Jergas M (2006) Effective dose of CT-and fluoroscopy-guided perineural/ epidural injections of the lumbar spine: a comparative study. Cardiovasc Intervent Radiol 29:84-91

\section{Publisher's Note}

Springer Nature remains neutral with regard to jurisdictional claims in published maps and institutional affiliations.

\section{Submit your manuscript to a SpringerOpen ${ }^{\circ}$ journal and benefit from:}

- Convenient online submission

- Rigorous peer review

- Open access: articles freely available online

- High visibility within the field

- Retaining the copyright to your article

Submit your next manuscript at springeropen.com 